\title{
Simulation of the impact of dangerous road incidents on driver's loss of time on express roads
}

\author{
Barbara Strzebrakowska, ${ }^{1, *}$ \\ ${ }^{1}$ Gdansk University of Technology, Faculty of Civil and Environmental Engineering, Gdansk, Poland
}

\begin{abstract}
Incidents on roads are an important factor in traffic flow disruption and a consequent loss of time for drivers. Disruptions can be the result of unexpected road incidents or planned repair works after an incident. These situations differ in the availability of information about the incidents and the resulting rerouting undertaken by drivers. The first part of the paper presents a literature review on the impact of road incidents on traffic and driver's loss of time modelling. Statistical analysis of the database of road incidents on motorways and express roads in the region of Pomorskie was conducted. The average time of arrival of emergency services to a scene and the average time of the rescue action were determined. Simulation research on road incidents was performed for different scenarios with modifications of traffic volume, duration of the road incidents and the degree of road section capacity limitations in various options. The simulation was performed by PTV VISUM software, with the DUE (Dynamic User Equilibrium) module for dynamic traffic modelling. The result of the research is the estimation of the average loss of time for the different variants of the incident, which enabled building a model of the driver's loss of time for specified event parameters.
\end{abstract}

\section{Introduction}

On their daily commute, road users choose their means of transport, the route and the time so that the journey is the most efficient in terms of duration, convenience and expense. With time drivers use the best route variant, which results in the traffic reaching equilibrium meaning that no other decision would result in a lower cost for drivers' journey [1]. The network is in a condition of equilibrium as a typical state, that contrary to appearances is very rare. Changes in traffic conditions occur frequently, caused by planned events such as holidays or mass events. The relevant information can be distributed by different methods so that drivers have a chance to change the route, means of transport or decide not to go. In the event of an unexpected incident such as an accident or collision it is impossible for drivers to change the route before setting off. Such events are unexpected and impossible to plan for [2].

\footnotetext{
* Corresponding author: barstrze@pg.gda.pl
} 


\section{Existing conditions}

\subsection{The impact of road incidents on traffic}

Road incidents can result in obstruction to normal passage on a section, interchange or junction, which involves a change in the parameters of traffic on the network. The character of this phenomenon is dynamic. All resulting actions and effects of an incident affect relevant interconnected parameters such as [3], section capacity and average speed of vehicles.

The decrease in capacity associated with the traffic incident depends on the number of lanes on the road and number of lanes blocked as a result. The study conducted in 2002 [4] suggests, for example, that a block of one lane on a two-lane road in each direction causes a capacity reduction of about $68 \%$ in the direction in which there is a traffic incident. Table 1 shows the detailed results.

Table 1. Percentage capacity of the section during the road incident [4]

\begin{tabular}{|l|c|c|c|c|c|}
\hline \multirow{2}{*}{\begin{tabular}{c}
\multirow{2}{*}{ Type of restriction } \\
\cline { 2 - 6 }
\end{tabular}} & \multicolumn{5}{|c|}{ Capacity during incident (\%) } \\
\cline { 2 - 6 } & 1 lane & 2 lanes & 3 lanes & 4 lanes & 5 lanes \\
\hline Vehicles in emergency lane & 0.45 & 0.75 & 0.84 & 0.89 & 0.93 \\
\hline 1 blocked lane & 0.00 & 0.32 & 0.53 & 0.56 & 0.75 \\
\hline 2 blocked lanes & - & 0.00 & 0.22 & 0.34 & 0.50 \\
\hline
\end{tabular}

The main effect of reduced capacity is the loss of time of drivers who are indirectly affected by the incident. Loss of time is understood as the difference between total travel time during normal average conditions and total travel time during the incident until normal conditions are restored. The analysis looks at the same time, duration and area for both situations.

\subsection{Driver time loss modelling methods}

There are several methods to estimate the time loss for road users when an incident occurs on a developed road network. Time loss may be estimated for a particular case referring to the road network, traffic volume, incident duration and type. Macroscopic, mesoscopic and microscopic computer simulations can be used for the calculation. For the analysis of road users' time losses as a result of road accidents each of these methods can be used [5].

For example, time loss estimates with the use of macrosimulation means introducing relevant restrictions on properly calibrated network. These changes will contribute to a change in the distribution of traffic on the network and make it possible to determine the changes in traffic volume in each section. In addition, it will be possible to calculate the loss of time for road users who will benefit from an alternative route bypassing the section where the incident took place. 


\section{Statistical analysis of road incidents}

Databases used in the paper relate to traffic incidents that occurred in the years $2012-2015$ on the motorway and express roads in Pomorskie. This database was made available by the Regional State Fire Service in Gdansk. In total, in the analysed period there were 177 road incidents on road S6; 55 incidents on the S7 and 166 on the A1 motorway [6]. The most frequent arrival time was 6 to 10 minutes. While it seems quick, it still exceeds 5 minutes. On a large number of routes, it took from 11 to 15 minutes. It was verified that the type of traffic incident did not affect significantly the average arrival time to the incident scene [6]. Response arrival times to the scene of incidents are summarised in the graph in Figure 1.

The time to deal with the consequences of an incident differs a lot. Emergency services, in most cases, took 10 to 100 minutes. There were incidents in which the consequences were dealt with for over $3 \mathrm{~h}$. The time of incident resolution on the S7 did not take more than $1 \mathrm{~h}$ 30 minutes. In most cases, it took 20 to 40 minutes. Only $10 \%$ of the incidents required actions longer than an hour. Time of dealing with the consequences of traffic incidents on the A1 motorway in most cases lasted from 10 minutes to 2 hours. As many as $8 \%$ of incidents required interventions of firefighters lasting over 2 hours. On average firefighters' actions took about 30 minutes [6].
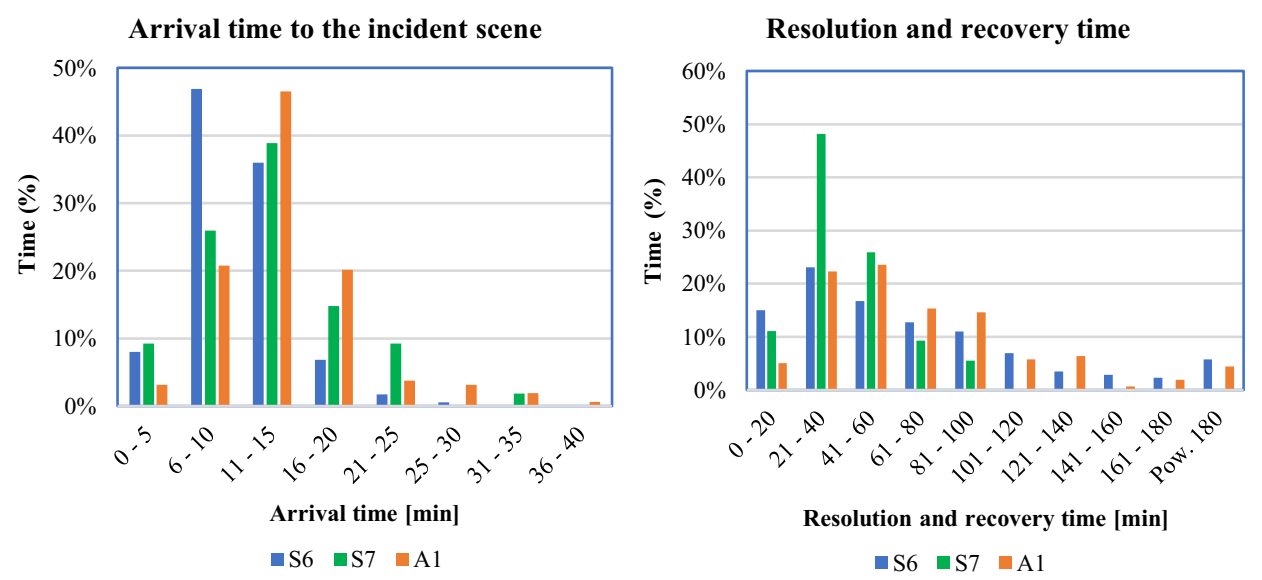

Fig. 1. Time of response arrival and resolving consequences of the incident (own study based on [6]).

\section{Simulation studies}

\subsection{Assumptions and variants of analysis}

To create a model of time loss caused by traffic incidents the analysis was performed for a section of the highway, with the parameters adopted as in the model for the Metropolitan Area of Gdansk, Gdynia and Sopot in which link capacities and section resistance function are based on measurements performed by University of Technology employees. The simulations were conducted for various parameters, therefore the variants provided, differed within such parameters and values as in Table 2. 
Tab. 2. The values of the parameters included in the model (own study).

\begin{tabular}{|c|c|}
\hline Parameter & Analysed scenarios \\
\hline Traffic volume & $500,1000, \ldots, 3500,4000,4400[\mathrm{P} / \mathrm{h}]$ \\
\hline Incident duration & $10 \mathrm{~min}, 20,40, \ldots, 160,180[\mathrm{~min}]$ \\
\hline Level of capacity reduction & $25 \%, 68 \%, 98 \%[4]$ \\
\hline
\end{tabular}

\subsection{The results of simulation studies}

The driver's time loss was obtained after conducting the simulation for all the variants of traffic volume and duration of the traffic incident for a blocked emergency lane. The resulting data were mapped in the graphs presented in Figure 5. The graphs show loss of time depending on the duration of the incident (the $\mathrm{X}$ axis) for different traffic volumes (the individual curves of the graph).

A traffic incident in which a vehicle or vehicles occupy an emergency lane results in speed reduction and limited lane capacity (25\%). Although traffic movement is still possible on two lanes, this movement is hampered. For traffic below $3000 \mathrm{veh} / \mathrm{h}$ time loss is insignificant: 0 - $10 \mathrm{veh} / \mathrm{h}$ in total for all drivers. If the traffic volume is close to the section's capacity, time loss values begin to grow rapidly. This is due to the fact that the queue which was formed, started dispersing slowly. This applies to a traffic volume with a capacity of over $80 \%$.

A traffic incident, in which one lane is blocked takes place when it is not possible to remove vehicles from the road onto the shoulder or emergency lane, or when it is possible to remove the vehicle from two lanes to one, so the traffic moves on one lane. In such cases, the maximum capacity of $68 \%$ was assumed. For traffic volume below $1000 \mathrm{veh} / \mathrm{h}$ time loss is insignificant: $0-12 \mathrm{veh} / \mathrm{h}$ in total for all drivers. If the traffic volume is greater than 1000 $\mathrm{veh} / \mathrm{h}$, the time loss values grow rapidly. This applies to a traffic volume with capacity as high as $34 \%$.

A traffic incident in which two lanes are blocked occurs when it is not possible to remove vehicles from the roads to allow movement on one lane or move the vehicles to the emergency lane or shoulder. Also, before removing the vehicles from the road, two lanes are blocked. The model assumes that capacity is reduced by $100 \%$. Even for a volume of about $500 \mathrm{veh} / \mathrm{h}$, the blockage on the entire cross-section of the road causes a considerable loss of time. The greater the traffic volume, the quicker the time loss grows. The queue of vehicles, which is formed as a result of the traffic incident disperses over a very long time. This applies to the traffic volume with a capacity of even $11 \%$.
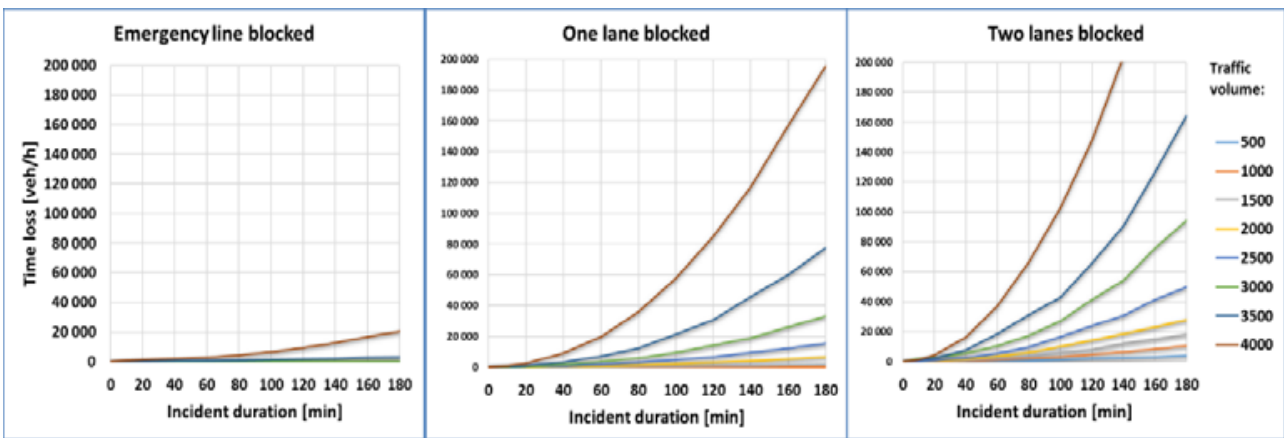

Fig. 3. Time loss graph - reduction variants of $25 \%, 68 \%$ and $100 \%$ (own study). 


\subsection{Time loss per vehicle}

Time loss per one vehicle for the variant of a blocked emergency lane is not more than 33 minutes, for the analysed scenarios. If the traffic volume is less than $3000 \mathrm{veh} / \mathrm{h}$ the time loss is almost zero, and amounts up to 1 minute. For higher traffic volumes, time loss increases to the point of becoming an impediment for the road users.

If one lane is blocked, the graph differs from the previous variant. For traffic over 1000 $\mathrm{veh} / \mathrm{h}$, the loss of time per vehicle begins to grow rapidly. With a further increase in traffic volume, time loss increases to a lesser extent. This is due more to the specifics of the behaviour of the queue of vehicles. The duration of the incident was observed to have a major impact.

If the entire cross-section of the road is blocked, the time loss graph per driver differs from the two preceding variants. In this case, the main factor of time loss is the duration of the traffic incident. It was observed that in every case, the time loss did not exceed the duration of the incident. If the traffic was at capacity, the road users' time loss would amount to the equivalent of the traffic incident duration. The increase in the time loss, per driver, depending on the traffic volume is of very low importance, but the loss is characterised by a modest increase. The graph of time losses for the various variants are shown in Figure 4.
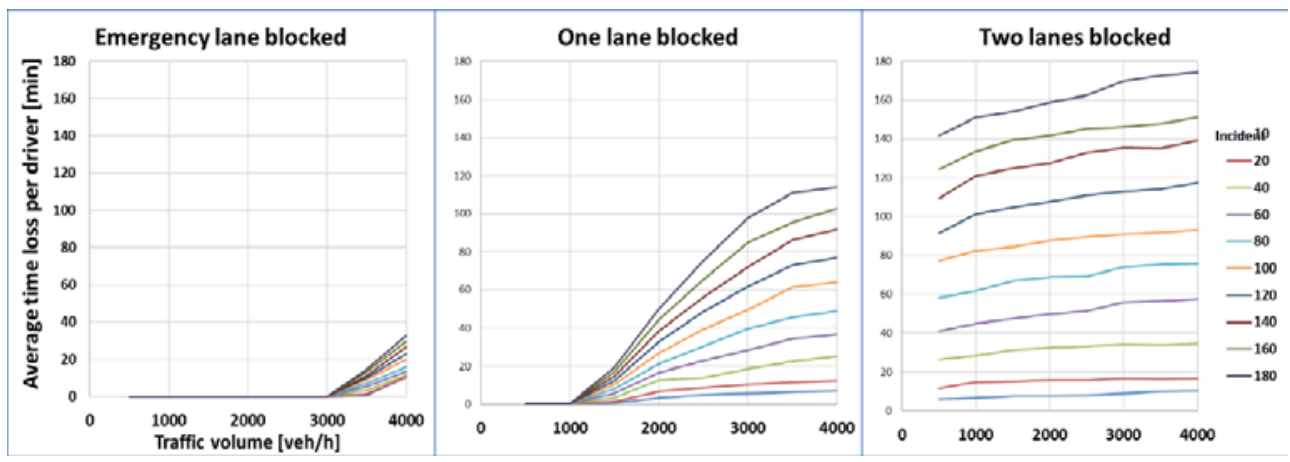

Fig 4. The graphs of average time loss per driver (own study).

\section{Time loss model}

The analysis and simulation study results made it possible to create a model of time loss depending on the parameters included in the analysis. The model of time loss is a function dependent on the traffic volume on the section, incident duration and level of capacity reduction. In addition to this, there are more factors that affect time loss significantly, which may also be added to the time loss model after further analysis.

The graph of function is variable depending on whether the reduction of capacity results in a so-called "bottleneck", hence causing the phenomenon of choking. If traffic volume is high and the reduction is significant it may cause an increase in the length of the queue which will be almost equal to the traffic volume. Long-lasting traffic incidents with such a reduction generate a significant increase in loss of time. The general function can be shown as:

$$
t_{s}=a \cdot t_{z}^{b} \cdot e^{(c \cdot s \cdot N)}
$$

where:

- $t_{s}$ - time loss,

- $N$ - volume,

- $t_{z}$ - incident duration, 
- $S$ - level of capacity reduction (relative decrease in capacity),

- $C$ - base section capacity in no incident situation $[\mathrm{veh} / \mathrm{h}]$,

$-a, b, c$ - coefficients.

\section{Conclusions}

\subsection{Summary}

Based on the analysis, it was found that it is possible to map the phenomenon of road users' time loss caused by a traffic incident, using a mathematical model.

It was noted that the most important parameters affecting the total time loss was traffic volume and the level of capacity reduction. These parameters are closely related and have a similar effect on the upward trend in time loss with increasing duration of the traffic incident. A different pattern of time loss increase is related to the extent of the restriction, the nature of the queue and the time needed to restore the traffic conditions to the state before the incident. Due to these differences, the time loss in the model is calculated using three formulas for different levels of restriction.

Loss of time per driver depends on the level of capacity reduction, traffic volume and duration of the traffic incident. The most important factor is capacity reduction because it alters the behaviour of the queue of vehicles. With the increase in restrictions, average time loss rises constantly, though to a decreasing extent. The maximum time loss per driver in given conditions will not be greater than the duration of the incident.

Due to the high importance of the level of capacity reduction for time loss, it is important to remove vehicles from the road or move vehicles to one lane as soon as possible to make at least one lane passable.

\subsection{Future research}

The proposed model of time loss is rather simplified in spite of the many variables. It refers to a situation where a driver cannot change the route. The analysed section was also simplified to one road with unchangeable cross-section and parameters.

Future research is expected to develop a model with more parameters which will allow a mapping of each traffic incident and estimating the time loss for a particular case. In addition to increasing the number of analysis parameters, it will provide a calculation of the additional variables dependent on the calculated time loss and resulting difficulties, such as increased fuel consumption or increased environmental pollution.

The analysis based on a simulation study is expected to be extended by adding field research and statistical analysis of the road incident database with greater detail than the study presented in the paper. The extended model will also be confronted and calibrated with actual data from national roads.

\section{References}

1. R. Kucharski, G. Gentile, Modelowanie zjawiska zmiany trasy przejazdu w dynamicznym rozkładzie ruchu w sieci drogowej, in: Res. Tech. J. Polish Assoc. Transp. Eng. Cracow, (Cracow, Poland, 2014)

2. R. Kucharski, Rerouting phenomena modelling for unexpected events in Dynamic Traffic Assignment. Modelowanie zjawisk zmiany trasy przejazdu w sytuacjach nietypowych, Cracow University of Technology, 2015 
3. PB Farradyne, Traffic incident management handbook (2000)

4. E. Chin, O. Franzese, D. Greene, H. Hwang, R. Gibson, Temporary losses of highway capacity and impacts on performance (2002)

5. S. Gaca, W. Suchorzewski, M. Tracz, Road traffic engineering. Theory and practice. Inżynieria ruchu drogowego. Teoria i praktyka. (WKŁ, Warsaw, 2008)

6. The database of the Pomeranian Voivodship Fire Service, (2016) 